\title{
Performance Analysis of Space-Time Codes with Channel Information Errors
}

\author{
Zhifeng Diao, Dongxu Shen, Victor O.K. Li \\ Department of Electrical and Electronic Engineering \\ The University of Hong Kong, Hong Kong, China
}

\begin{abstract}
Many space-time codes (STC) have been proposed to enhance the performance of wireless communications in flat fading channels. All of them rely on the knowledge of the channel, and are hence affected by the channel estimation errors. Most previous research on STC performance evaluation assume perfect channel information. In this paper, we investigate STC robustness under imperfect channel knowledge. We first define the concept of "closeness" by comparing the BER under channel estimation errors with that of perfect channel knowledge, aiming to characterize STC performance degradation due to imperfect channel knowledge. Then the robustness of STC can be compared by their "closeness" to perfect results. In our computer simulations, we apply the same channel estimator to different STCs in Orthogonal Frequency Division Multiplexing (OFDM) communication systems. We find that for systems with two and three transmit antennas, the space time block codes (STBC) are always more robust to channel estimation errors than space time trellis codes (STTC). With the increase of receive diversity, all STCs become more robust to the channel estimation errors. For STTC, as the number of trellis states increases, the codes become less robust to the channel estimation errors. We also compare the BER performance of STC in the presence of channel estimation errors. For the two-transmitantenna system, the performance of STBC is always better than that of the 4-state STTC, but is always worse than 16-state STTC. For systems with three transmit antennas, the BER performance of STTC is much better than that of STBC.
\end{abstract}

\section{INTRODUCTION}

Recently, space-time codes (STC) have been proposed as an effective method for high data rate transmission over flat fading channels using multiple transmit antennas. STC can be generally divided into two types: trellis codes (STTC) and block codes (STBC). Tarokh et al. [1] proposed STTC with two transmit antennas. STTC for more transmit antennas are provided in [2]. In [3], Alamouti presented an STBC for two transmit antennas. STBC for more transmit antennas are generalized in [4].

OFDM is a promising technique for achieving high data rate and combating multipath fading in wireless communications by transforming a frequency selective fading channel into a number of parallel, low-rate, flat fading sub-channels. Thus OFDM is suitable for STC implementation [5].

All STC require the knowledge of the channel for decoding. Most previous evaluations of code performance assume perfect channel knowledge. In practice, channel information has to be estimated, and is never perfect. For OFDM systems with multiple transmit antennas, Y. Li et al. [6] developed a channel estimation algorithm. A simplified channel estimator was given in [7] using optimum training sequences.

In this paper, we evaluate the performance of STC in the presence of channel estimation errors. The channel estimation algorithm of Y. Li et al. [6] is employed in our simulation. With this channel estimator, we find that the performance of STC are affected differently. We define the STC "closeness" to perfect results by comparing the BER under channel estimation errors with that of perfect channel knowledge. Then the STC robustness to channel estimation errors can be represented by its closeness to perfection, i.e., an STC is more robust if it is closer to the perfect result. By computer simulation, we find that STBC is always more robust to the channel estimation errors than STTC regardless of the number of transmit antennas. With the increase of receive diversity, all STCs become more robust to channel estimation errors. For STTC, as the number of states increases, the robustness decreases. On the other hand, the BER of all STC in the presence of channel estimation errors are also compared. In systems with two transmit antennas, the BER performance of STBC is better than 4-state STTC, but worse than 16-state STTC. With three transmit antennas, the BER of STTC is much better than STBC.

This paper is organized as follows. In Section 2, we introduce the channel model and the channel estimator. In Section 3, the impacts of channel estimation errors on STTC and STBC are analyzed. Simulation results are given in Section 4. Section 5 is the conclusion.

\section{BACKGROUND AND SYSTEM DESCRIPTION}

We consider an OFDM system with $N_{T}$ transmit antennas and $N_{R}$ receive antennas. The wireless channels between transmit antennas and the receive antennas are assumed to be independent.

\section{A. Channel Model}

We assume the multipath fading channel is wide sense stationary with uncorrelated scattering. With tolerable leakage [6], the time domain channel impulse response is modeled as a tapped delay line at a tap spacing of a sampling interval. The channel impulse response can be expressed as

$$
h(t, \tau)=\sum_{i=1}^{P} a_{i}(t) \delta\left(t-\tau_{i}\right)
$$


where $a_{i}(t)$ is the complex gain of path $i, \tau_{i}$ is the corresponding path delay, $P$ is the number of paths, and $\delta(\tau)$ is the Dirac delta function. Here $\tau_{i}=i \Delta t$, where $\Delta t$ is the sampling interval of the OFDM system. We assume the channel in an STC block is constant.

In the frequency domain, the channel response is represented as

$$
H(n, j)=\sum_{i=1}^{P} a_{i}(n T s) W^{i j}
$$

where $n$ is the index for an OFDM symbol, $j$ is the subcarrier index, $T_{s}$ is the duration of an OFDM symbol, $W=e^{-j 2 \pi / N_{c}}$, and $N_{c}$ is the number of OFDM subcarriers.

\section{B. Channel Estimator}

In this paper, we adopt the channel estimator in [6]. Let $E_{0}$ be the symbol energy on a pilot subcarrier. We define the pilot signal to noise ratio (PSNR) on a pilot subcarrier as

$$
\gamma=\frac{E_{0}}{\sigma_{0}^{2}}
$$

where $\sigma_{0}^{2}$ is the average noise power. According to the analysis in [6], the channel estimation error on a channel tap in the time domain has variance $\frac{1}{\gamma}$ when the optimal pilot sequence is adopted. Then the variance of channel estimation error on any OFDM subcarrier is obviously solely determined by PSNR.

\section{Characterization AND AnAlysis OF ChanNEL ESTIMATION ERRORS ON STC}

Space-time codes can be generally divided into two classes: STTC and STBC. We first propose the concept of STC closeness to perfect results and STC robustness, then analyze the impacts of channel estimation errors on STTC and STBC.

\section{A. Closeness and Robustness}

With fixed SNR, the channel estimation error is reduced as PSNR increases, and the BER performance should be closer to that with perfect channel information. So the influence of channel estimation errors can obviously be represented by the difference between the BER under channel estimation error and the perfect one.

Let $e_{n}$ be the BER with non-perfect channel information, $e_{p}$ be the BER with perfect channel information. We define STC closeness to perfection as

$$
\Phi=10 \log \frac{e_{n}}{e_{p}}
$$

The concept "closeness" characterizes the impact of channel estimation errors on the performance of STC.

The robustness of an STC can be represented by its closeness, i.e., an STC is more robust than another if it is closer to perfection. In other words, a smaller $\Phi$ means more robustness.

Here we should note the concept of robustness only represent the sensitivity of an STC to channel estimation errors. However, robustness is different from BER performance. It is possible that STC "A" is more robust than "B", but A still has worse BER than B.

\section{B. STTC under Channel Estimation Error}

In an STTC encoder, the data stream is mapped into $N_{T}$ streams of symbols which are drawn from a signal constellation of size $2^{b}$, where $b$ is the number of bits per symbol. At the receiver, the Viterbi algorithm is used to compute the path with the lowest accumulated metric. The branch metric is calculated based on the channel estimation results. The signal at the $n_{r} t h$ receiver antenna can be presented as

$$
r_{l}^{n_{r}}=\sum_{n_{t}=1}^{N_{T}} H_{l, n_{t}}^{n_{r}} x_{l, n_{t}}+\eta, l=1,2 \cdots L, n_{r}=1,2 \cdots N_{R}
$$

where $L$ is the length of the input bit per decoding interval, and $x_{l, n_{t}}$ is the complex valued modulation symbol transmitted from the $n_{t}$ th transmit antenna in the $l t h$ symbol interval and $H_{l, n_{t}}^{n_{r}}$ is the channel response between the $n_{t} t h$ transmit antenna and the $n_{r}$ th receive antenna. With an estimated channel, the maximum likelihood decoder [1] intends to minimize the metric.

$$
\begin{aligned}
F(r, x) & =\Sigma_{l=1}^{L} \Sigma_{n_{r=1}}^{N_{R}}\left|r_{l}^{n_{r}}-\sum_{n_{t}=1}^{N_{T}} \widehat{H}_{l, n_{t}}^{n_{r}} x_{l, n_{t}}\right|^{2} \\
& =\Sigma_{l=1}^{L} \Sigma_{n_{r=1}}^{N_{R}}\left|r_{l}^{n_{r}}-\sum_{n_{t}=1}^{N_{T}}\left(H_{l, n_{t}}^{n_{r}}+e_{n_{t}}^{n_{r}}\right) x_{l, n_{t}}\right|^{2}
\end{aligned}
$$

where $\widehat{H}_{l, n_{t}}^{n_{r}}=H_{l, n_{t}}^{n_{r}}+e_{n_{t}}^{n_{r}}$ is the estimated channel response, $e_{n_{t}}^{n_{r}}$ is the corresponding channel estimation error.

It is well known that the errors in the output of the Viterbi decoder are in bursts, and can not be assumed as uniformly distributed. An approximation of the error probability can be calculated by [8]

$$
P_{b} \approx \frac{1}{L} \sum_{j} m_{i j} P(x \rightarrow \widehat{x})
$$

In (7), $m_{i j}$ is the length of the bit-error burst associated with each error event, and the pairwise error probability $P(x \rightarrow \widehat{x})$ is the probability of confusing the codeword $x$ with the codeword $\widehat{x}$. According to [9], $P(x \rightarrow \widehat{x})$ is given by

$$
P(x \rightarrow \widehat{x})=\operatorname{Pr}[F(r, \widehat{x}) \leq F(r, x)]=\operatorname{Pr}[D \leq 0]
$$

where $D$ is defined as

$$
\begin{aligned}
D= & \sum_{l=1}^{L} \sum_{n_{r}=1}^{N_{R}} D_{l}^{n} \\
= & \sum_{l=1}^{L} \sum_{n_{r}=1}^{N_{R}}\left\{\left|r_{l}^{n_{r}}-\sum_{n_{t}=1}^{N_{T}} \widehat{H}_{l, n_{t}}^{n_{r}} \widehat{x}_{l, n_{t}}\right|^{2}\right. \\
& \left.-\left|r_{l}^{n_{r}}-\sum_{n_{t}=1}^{N_{T}} \widehat{H}_{l, n_{t}}^{n_{r}} x_{l, n_{t}}\right|^{2}\right\}
\end{aligned}
$$


By inserting (5), $D_{l}^{n}$ is given as

$$
\begin{aligned}
D_{l}^{n_{r}}= & \mid \sum_{n_{t}=1}^{N_{T}} H_{l, n_{t}}^{n_{r}}\left(x_{l, n_{t}}-\widehat{x}_{l, n_{t}}\right)+\eta \\
& -\left.\sum_{n_{t}=1}^{N_{r}} e_{n_{t}}^{n_{r} \widehat{x}_{l, n_{t}}}\right|^{2}-\left|\eta-\sum_{n_{t}=1}^{N_{r}} e_{n_{t}}^{n_{r}} x_{l, n_{t}}\right|^{2} \\
= & \left|\sum_{n_{t}=1}^{N_{T}} H_{l, n_{t}}^{n_{r}}\left(x_{l, n_{t}}-\widehat{x}_{l, n_{t}}\right)+\eta_{a}\right|^{2}-\left|\eta_{b}\right|^{2}
\end{aligned}
$$

where $\eta_{a}=\eta-\sum_{n_{t}=1}^{N_{r}} e_{n_{t}}^{n_{r}} \widehat{x}_{l, n_{t}}$ and $\eta_{b}=\eta-\sum_{n_{t}=1}^{N_{r}} e_{n_{t}}^{n_{r}} x_{l, n_{t}}$. From equation (10), it is found that the channel estimation error can be regarded as the increase of noise, which deteriorates the pairwise error probability.

\section{STBC under Channel Estimation Error}

Alamouti [3] presented a simple STBC with two transmit antennas. STBC constructions for 3 and 4 transmit antennas were given in [4].

We only analyze the impact of channel estimation errors on Alamouti's scheme with one receive antenna. The analysis for other STBC is similar. In OFDM systems, the STBC encoding operation is carried out on the same subcarriers of two consecutive OFDM symbols. Let $S_{0}$ and $S_{1}$ be the data symbols in the same subcarrier location of two consecutive OFDM symbols. For the encoding scheme, the symbols $S_{0}$ and $S_{1}$ are transmitted at time $k$ from transmit antennas 1 and 2 respectively. Then at time $k+1,-S_{1}^{*}$ is transmitted from antenna 1 and $S_{0}^{*}$ from antenna 2 , where $*$ denotes the complex-conjugate operation. Based on channel estimation, the receiver decodes the received signal by the maximum likelihood decision rule.

Let $h_{0}, h_{1}$ be the channel response between the two transmit antennas and the receive antenna. Let $e_{0}$ be the channel estimation error of $h_{0}$ and $e_{1}$ be the channel estimation error of $h_{1}$. The received signals at time $k$ and $k+1$ are :

$$
\begin{aligned}
& r_{0}=h_{0} S_{0}+h_{1} S_{1}+\eta_{0} \\
& r_{1}=-h_{0} S_{1}^{*}+h_{1} S_{0}^{*}+\eta_{1}
\end{aligned}
$$

where $\eta_{0}$ and $\eta_{1}$ are complex Gaussian noise.

After combining,

$$
\begin{aligned}
& \widehat{S_{0}}={\widehat{h_{0}}}^{*} r_{0}+\widehat{h_{1}} r_{1}^{*} \\
& \widehat{S_{1}}={\widehat{h_{1}}}^{*} r_{0}-\widehat{h_{0}} r_{1}^{*}
\end{aligned}
$$

where $\widehat{h_{0}}, \widehat{h_{1}}$ are the estimation result of $h_{0}, h_{1}$. Substituting (11) into (12), together with $\widehat{h_{0}}=h_{0}+e_{0}, \widehat{h_{1}}=h_{1}+e_{1}$, we obtain

$$
\widehat{S_{0}}=\left(\left|h_{0}\right|^{2}+\left|h_{1}\right|^{2}\right) S_{0}+h_{0}^{*} \eta_{0}+h_{1} \eta_{1}^{*}+r_{0} e_{0}^{*}+r_{1}^{*} e_{1}
$$

From (13), we can see that the channel estimation error increases the channel noise, as given by $n^{\prime}=h_{0}^{*} \eta_{0}+h_{1} \eta_{1}^{*}+$ $r_{0} e_{0}^{*}+r_{1}^{*} e_{1}$. Without loss of generality, we assume that the channel has unit power, i.e., $E\left(h_{i} h_{i}^{*}\right)=1, i=0,1$. We denote $E_{s}=E\left(S_{i} S_{i}^{*}\right), i=0,1$. Since the power of $\eta_{i} e_{j}, i, j=0,1$ is relatively small, it can be neglected. With equation (3), the variance of $n^{\prime}$ is calculated as

$$
\begin{aligned}
\sigma_{n_{e}}^{2} & =2 \sigma_{0}^{2}+4 E_{s} \sigma_{e}^{2} \\
& =2 E_{s}\left(\chi^{-1}+g\left(\gamma^{-1}\right)\right)
\end{aligned}
$$

where $\chi=\frac{E_{s}}{\sigma_{0}^{2}}$ is the signal-to-noise ratio (SNR) of the data subcarrier. The second term of (14) is the noise caused by the channel estimation error, which is a function of $\gamma$. As PSNR increases, the noise caused by channel estimation error decreases, and performance will be closer to that with perfect channel knowledge. The robustness of STBC is thus determined by the term $g\left(\gamma^{-1}\right)$. Then the SNR for $\widehat{S_{0}}$ is given by

$$
\begin{aligned}
\Gamma & =\frac{E\left\{\left(\left|h_{0}\right|^{2}+\left|h_{1}\right|^{2}\right)^{2}\right\} E_{s}}{2 E_{s}\left(\chi^{-1}+g\left(\gamma^{-1}\right)\right)} \\
& =\frac{E\left\{\left(\left|h_{0}\right|^{2}+\left|h_{1}\right|^{2}\right)^{2}\right\}}{2\left(\chi^{-1}+g\left(\gamma^{-1}\right)\right)}
\end{aligned}
$$

Equation (15) shows that $\Gamma$ depends on both the data SNR and PSNR. In other words, the increase of $\Gamma$ can only be achieved by the simultaneous increase of SNR and PSNR.

The above analysis can be easily extended to cases of more than one receive antenna and other block STC.

\section{Simulation Results}

We simulate STC-OFDM systems with 2 and 3 transmit antennas and 1 or 2 receive antennas. The OFDM system has 64 subcarriers occupying $20 \mathrm{MHz}$ bandwidth at $5.4 \mathrm{GHz}$ with 6 guard subcarriers at each end. Each OFDM symbol lasts for a duration of $4 \mu \mathrm{s}$, in which $0.8 \mu \mathrm{s}$ is the guard interval. The sampling period is $0.05 \mu \mathrm{s}$. We consider the quasi-static multipath Rayleigh fading channel. The multipath channel for each antenna has 6 taps of Rayleigh faded paths at an interval $0.05 \mu \mathrm{s}$, and the power delay profile follows an exponential decay rule of $\left[1, e^{-1}, e^{-2}, e^{-3}, e^{-4}, e^{-5}\right]$. In the simulation, the transmit power is equally divided on transmit antennas.

\section{A. Robustness Comparison}

We consider 4-, 8- and 16-state STTC and STBC with quadrature-phase shift keying (QPSK) modulation in twotransmit- antenna systems [1].

Fig. 1 shows the BER performance of 4-state STTC with one and two receive antennas with fixed PSNR. As PSNR increases, the performance is closer to that with perfect channel knowledge. With one receive antenna, only when the PSNR is $30 \mathrm{~dB}$, the performance curve is close enough to the perfect one. With two receive antennas, for the same PSNR, the curve is closer to that of perfect.

Fig. 2 illustrates the BER performance of STTC (4, 8, 16 states) and STBC with respect to PSNR in a two-transmitantenna and one-receive-antenna system. The data SNR is fixed 
at $30 \mathrm{~dB}$. With the increase of PSNR, the BER performance is closer to the perfect one. However, it is not easy to compare the STC robustness to channel estimation errors from Fig. 1 and Fig. 2.

Based on the results in Fig. 2, the robustness of STTC and STBC can be easily compared. Using the concept of "closeness," we find from Fig. 3 that for the same closeness, STBC requires the lowest PSNR, followed by 4-state STTC, 8 -state STTC and 16-state STTC. Therefore, STBC is the most robust to channel estimation errors and 16-state STTC is the least. Thus, as the number of the trellis states increases, the robustness of STTC is reduced.

Fig. 4 shows the robustness of STC in systems with two transmit and two receive antennas. We observe that STBC is still the most robust to channel estimation errors. Similar to Fig. 3, the robustness of STTC is also reduced as the number of trellis states increases.

For OFDM systems with three transmit antennas, the STBC transmit matrix is provided in [4], with a coding rate $1 / 2$; for STTC, the connection polynomial is given in [2] as $(1+2 D+$ $\left.2 D^{2}, 2+D+2 D^{2}, 2+2 D+D^{2}\right)$, with a coding rate 1 . To fairly compare the robustness of the two STC schemes, 16QAM modulation is applied in STBC, while QPSK modulation is adopted in STTC, so that the two schemes have the same information bit rate. The closeness of STBC and STTC are given in Fig. 5. The SNR is fixed at 20dB. By comparison, regardless of the number of receive antennas, STBC is always more robust than STTC. Both STBC and STTC are more robust to channel estimation errors with two receive antennas than with one receive antenna.

\section{B. BER Performance Comparison}

In practice, it is convenient to assign the same energy on pilot and data subcarriers. We thus set SNR equal to PSNR. Fig 6 shows the BER performance curve of all STC in systems with two transmit antennas. For systems with 2 transmit and 1 receive antenna, STBC outperforms 4-state STTC, 8-state STTC, but it is inferior to that of 16-state STTC. For systems with 2 transmit and 2 receive antennas, STBC can only outperform the 4-state STTC. The 16-state STTC is still the best in performance. For three-transmit-antenna systems, on the contrary, as shown in Fig. 7, the performance of STTC is much better than that of STBC. Admittedly, 16-state STTC is the most complex among the evaluated schemes, and the performance enhancement is at the expense of additional complexity.

The above results show the robustness and BER performance are different. An STC that is robust does not necessarily have the best BER performance.

\section{CONCLUSION}

In this paper, the concept of STC closeness to perfect results is introduced, based on which robustness of STC can be reflected. From the robustness comparison, we find that STBC is always more robust to channel estimation errors than STTC. With more receive diversity, the robustness is also

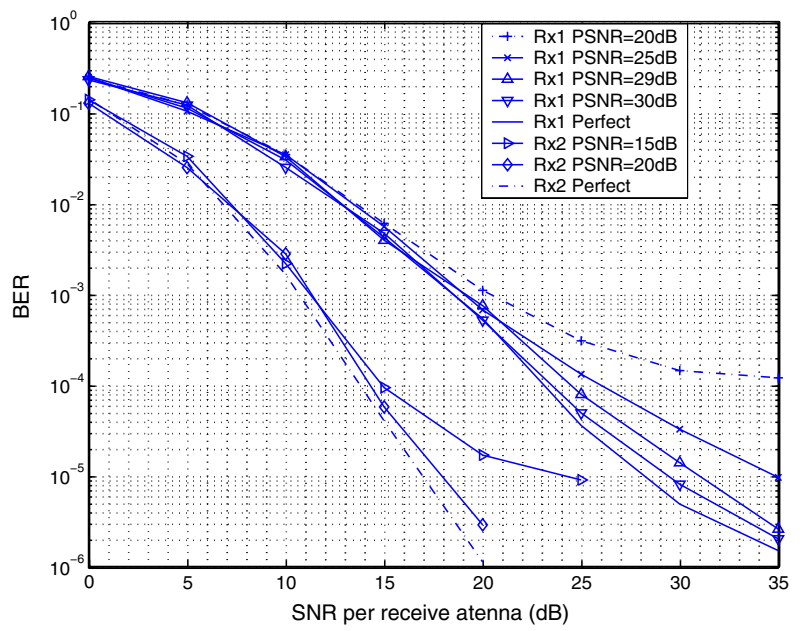

Fig. 1. Performance of 4-state STTC with fixed PSNR in systems with one and two receive antennas.

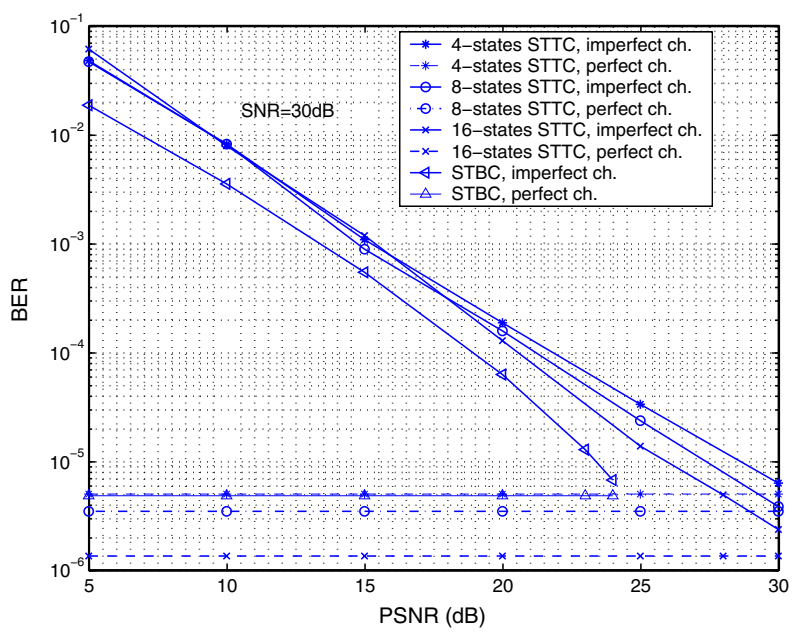

Fig. 2. BER performance of STTC and STBC in a Tx2Rx1 system with fixed SNR and varying PSNR.

increased. For STTC, as the number of trellis states increases, the robustness to channel estimation errors is reduced. On the other hand, the BER performance of all STC are also compared in the presence of channel estimation errors. In two-transmitantenna systems, the BER of STBC is better than 4-state STTC, but worse than 16-state STTC. In three-transmit-antenna systems, the performance of STTC is much better than STBC.

\section{ACKNOWLEDGEMENT}

This research is supported in part by the Research Grants Council of the Hong Kong Special Administration Region, China (Project No. HKU 7047/00E).

\section{REFERENCES}

[1] V. Tarokh, N. Seshadri, and A.R. Calderbank, "Space-time codes for high data rate wireless communication: Performance analysis and code construction," IEEE Trans. Inform. Theory, vol. 44, pp.744-765, Mar. 1998. 


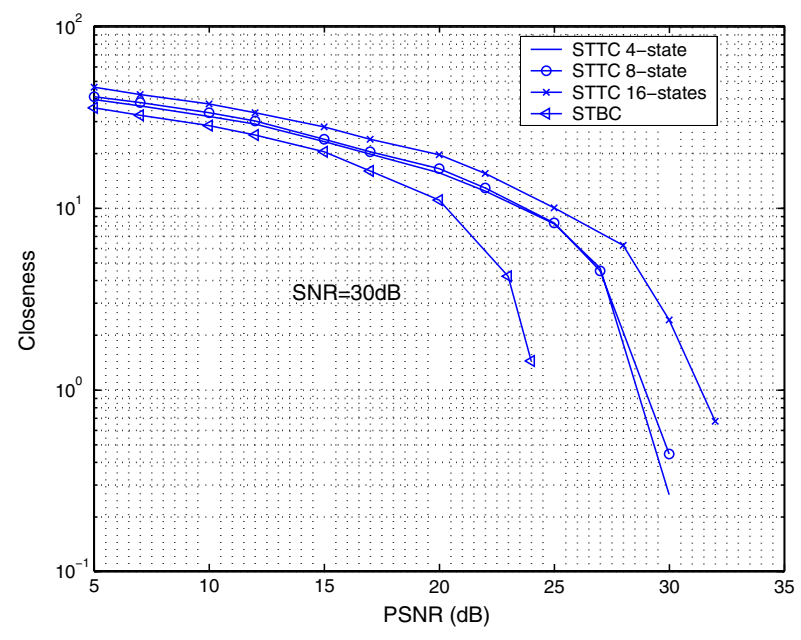

Fig. 3. Comparison of STC robustness in a Tx2Rx1 system

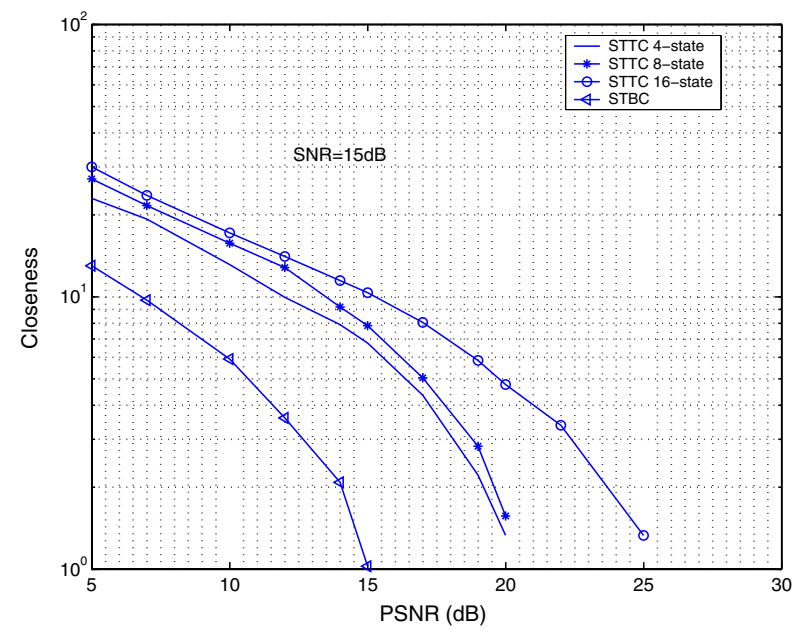

Fig. 4. Comparison of STC robustness in a Tx2Rx2 system

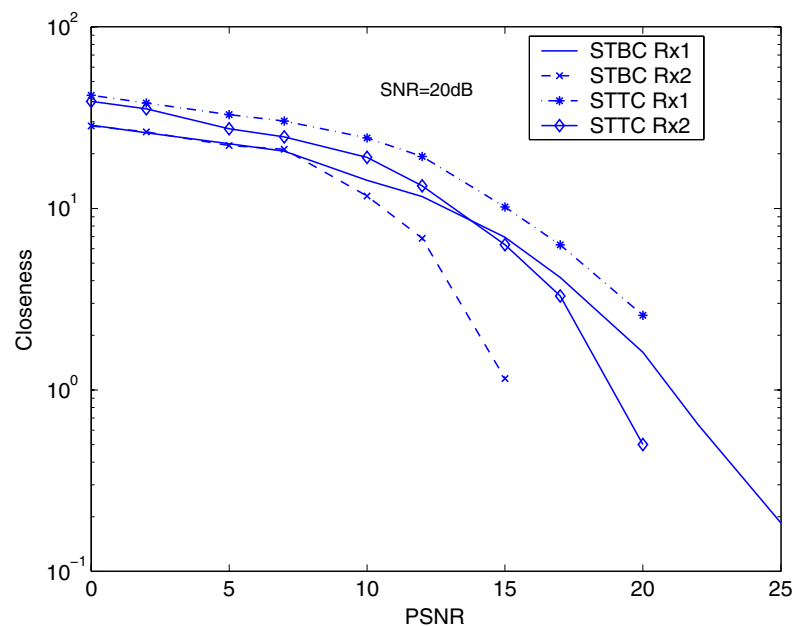

Fig. 5. Comparison of STC robustness in Tx3Rx1 and Tx3Rx2 systems

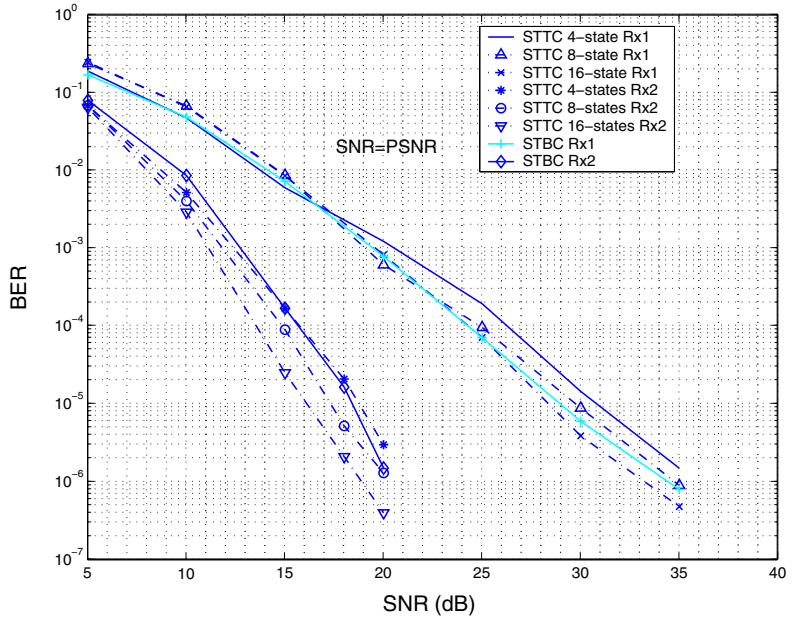

Fig. 6. Performance comparison between STTC and STBC with two transmit antennas

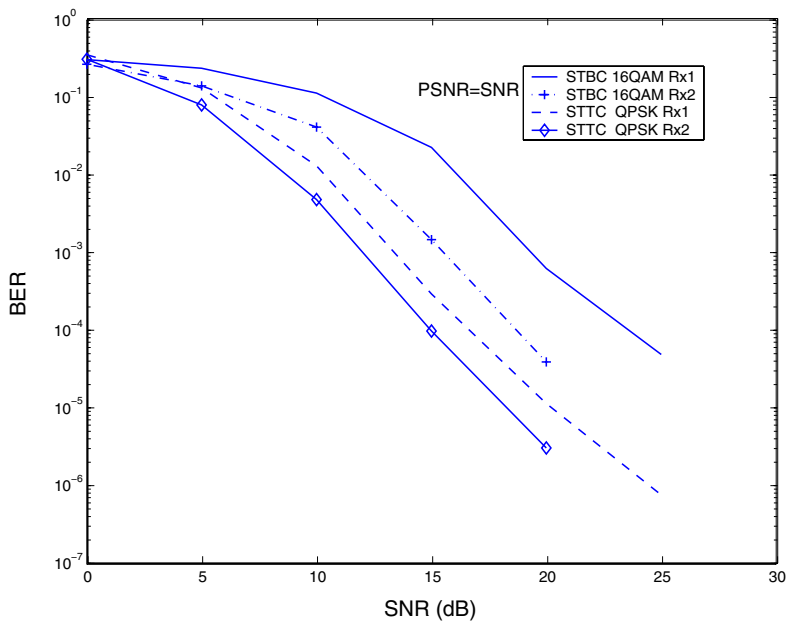

Fig. 7. Tx3 performance comparison

[2] H. E1 Gamal and A. R. Hammons, " On the design and performance of algebraic space-time codes for BPSK and QPSK modulation," IEEE Trans Commun.,vol 50, no.6, Jun. 2002.

[3] S. M. Alamouti, "A simple transmitter diversity scheme for wireless communications," IEEE J. Select. Areas Commun., vol. 45, pp. 1451-1458, July. 1999.

[4] V. Tarokh, H. Jafarkhani, and A.R. Calderbank, "Space-time block codes from orthogonal designs," IEEE Trans. Inform. Theory, vol. 44, pp.1456-1467, Mar. 1998.

[5] N. AL-Dhahir, et al., " Space-time processing for broadband wireless access," IEEE Commn Magazine, vol.40, no.9, pp. 136-142, Sep 2002.

[6] Y. Li, N. Seshadri and S. Ariyavisitakul, "Channel estimation for OFDM systems with Transmitter Diversity in mobile wireless channels," IEEE J. Select. Areas Commun., vol. 17, pp. 461-471, Mar. 1999.

[7] Y. Li, " Simplified channel estimation for OFDM systems with multiple transmit antennas, "IEEE Trans. Commun., vol. 1, no.1, Jan. 2002.

[8] J. K. Carvers. et al., "Analysis of the error performance of trellis-coded modulations in rayleigh-fading channels," IEEE Trans. Commun., vol. 40, No.1, pp. 74-83, Jan 1992.

[9] M. Uysal. et al., "Error performance analysis of space-time codes over Rayleigh fading channels," IEEE VTC-Fall 2000, vol. 5, pp. 2285-2290, Sept. 2000. 